\title{
Bounds on the value-at-risk for the sum of possibly dependent risks
}

\author{
Mhamed Mesfioui, Jean-François Quessy* \\ Département de Mathématiques et d'informatique, Université du Québec à Trois-Rivières, C.P. 500, \\ Trois-Rivières, Que., Canada G9A $5 H 7$
}

Received June 2004; received in revised form November 2004; accepted 14 March 2005

\begin{abstract}
In this paper, explicit lower and upper bounds on the value-at-risk $(\mathrm{VaR})$ for the sum of possibly dependent risks are derived when only partial information is available about the dependence structure and the individual behaviors. When the marginal distributions are known, a reformulation of a result of Embrechts et al. [Finan. Stoch. 7 (2003) 145-167] makes it possible, under some regularity conditions, to compute explicit bounds for the VaR under various dependence scenarios. In the case where only the means and the variances of the risks are available, explicit bounds are obtained from an optimization over all possible values of the correlation matrix associated with the vector of risks. Analytical and numerical investigations are presented in order to investigate the quality of these bounds.
\end{abstract}

() 2005 Elsevier B.V. All rights reserved.

Keywords: Comonotonicity; Copula; Fréchet-Hoeffding bounds; Correlation matrix; Value-at-risk

\section{Introduction}

In actuarial science and in finance, value-at-risk measures, at a predetermined confidence level and under normal market conditions, the worst loss that an institution can suffer over a given time period. In risk management, it is thus an important tool since it allows for the quantification of the volatility of a company's assets.

In theoretical terms, the value-at-risk (VaR) of a random variable at level $\alpha$ is simply defined as the $\alpha$ th quantile of its distribution. Due to its computational simplicity and for some regularity reasons, value-at-risk remains one of the most popular measures of risk despite the fact that it has been severely criticized for not being coherent. More specifically, the VaR is not sub-additive, and hence the risk associated to a given portfolio can be larger than the sum of the stand-alone risks of its components when measured by the VaR; see, e.g., Artzner et al. (1999). In order

\footnotetext{
* Corresponding author.

E-mail address: Jean-Francois.Quessy@uqtr.ca (J.-F. Quessy).
} 
to circumvent this problem, Embrechts et al. (2003) use the concept of copula to bound the value-at-risk of the sum of several risks when the marginal distributions are known.

The first purpose of this article is to provide explicit expressions for the bounds proposed by Embrechts et al. (2003) under various distributional assumptions. This is achieved at the price of some restrictions on the densities of the risks. The second goal of the paper is to derive bounds when only the first two moments of the risks are known.

The paper is organized as follows. The necessary definitions and notations are recalled in Section 2. Explicit expressions for the lower and the upper bounds are then obtained in Section 3 under some restrictions on the associated densities of the risks. In Section 4, the situation in which only the means and the variances of the risks are known is treated. A reformulation, in a value-at-risk context, of the univariate extreme distributions of Kaas and Goovaerts (1986) leads to lower and upper bounds for the value-at-risk of the sum of several risks by optimizing over all possible values of the associated correlation matrix. This method is shown to be efficient when compared to another approach using copula theory. Finally, Section 5 is devoted to the comparison of the various bounds encountered in the paper.

\section{Preliminaries}

The basic concepts and results to be used in the remaining of the paper are given below.

Definition 2.1. Let $\overline{\mathbb{R}}=\mathbb{R} \cup\{ \pm \infty\}$ be the extended real line and define inf $\emptyset=-\infty$. The generalized leftcontinuous inverse of a non-decreasing function $f: \mathbb{R} \rightarrow \mathbb{R}$ is the mapping $f^{-1}: \mathbb{R} \rightarrow \mathbb{R}$ defined by

$$
f^{-1}(t)=\inf \{s \in \mathbb{R} \mid f(s) \geq t\} .
$$

From this definition, one has that $f^{-1}$ is non-decreasing. In addition, if $f$ is right-continuous and $f^{-1}(t)<\infty$, then $f(s) \leq t$ implies that $f^{-1}(t) \geq s$.

Definition 2.2. Let $F$ be the (right-continuous) distribution function of a random variable $X$. Then, the value-at-risk of $X$ at level $\alpha$ is defined as

$$
\operatorname{VaR}_{\alpha}(X)=F^{-1}(\alpha)
$$

In many financial applications, one wants to evaluate the risk level of a portfolio of $n \geq 2$ possibly dependent risks. This calls for the study of the dependence structure among the risks. In modern dependence theory, this is accomplished via the use of copulas. For an excellent exposition, see Nelsen (1999). Formally, let the multivariate distribution function of a random vector $X=\left(X_{1}, \ldots, X_{n}\right)$ be defined as $H\left(x_{1}, \ldots, x_{n}\right)=\mathrm{P}\left(X_{1} \leq x_{1}, \ldots, X_{n} \leq\right.$ $x_{n}$ ), and denote by $F_{1}, \ldots, F_{n}$ its associated marginal distributions. The theorem stated next, due to Sklar (1959), enables $H$ to be linked with $F_{1}, \ldots, F_{n}$ through a distribution function $C:[0,1]^{n} \rightarrow[0,1]$ with uniform marginals, called a copula.

Theorem 2.1. If $H$ is a multivariate distribution function whose univariate marginals are $F_{1}, \ldots, F_{n}$, there exists a multidimensional copula $C$ such that $H\left(x_{1}, \ldots, x_{n}\right)=C\left\{F_{1}\left(x_{1}\right), \ldots, F_{n}\left(x_{n}\right)\right\}$ for all $x_{1}, \ldots, x_{n} \in \mathbb{R}$. If $F_{1}, \ldots, F_{n}$ are continuous, then $C$ is unique.

An interesting feature of copulas is that whenever the marginal distributions are continuous, $C$ contains all the information about the dependence structure of $\left(X_{1}, \ldots, X_{n}\right)$. For example, the theoretical value of de- 
pendence measures like Kendall's tau and Spearman's rho depend only on the copula underlying a given population.

The following definition gives the copula analogue of the notion of exchangeable random variables. This property is shared by members of many well-known families of copulas.

Definition 2.3. A copula $C$ is exchangeable if $C\left(u_{\tau(1)}, \ldots, u_{\tau(n)}\right)=C\left(u_{1}, \ldots, u_{n}\right)$ for any permutation $\tau$ of the set $\{1, \ldots, n\}$. When $n=2, C$ is said to be symmetric.

Now the definition of the dual associated to a given copula is provided. This notion will prove crucial throughout this work.

Definition 2.4. Let $C$ be the distribution function of a random vector $\left(U_{1}, \ldots, U_{n}\right)$ with uniform marginals. Then, the dual of $C$ is defined by

$$
C^{\mathrm{d}}\left(u_{1}, \ldots, u_{n}\right)=\mathrm{P}\left(\bigcup_{i=1}^{n}\left\{U_{i} \leq u_{i}\right\}\right)
$$

It is possible to order copulas by comparing them pointwise. Explicitly, let $C_{1}$ and $C_{2}$ be $n$-variate copulas such that for all $u=\left(u_{1}, \ldots, u_{n}\right) \in[0,1]^{n}$, the inequality $C_{1}(u) \leq C_{2}(u)$ holds. It is then said that $C_{1}$ is smaller than $C_{2}$, termed $C_{1} \leq C_{2}$. A useful result is that any copula $C$ lies between the lower and upper Fréchet-Hoeffding bounds. Specifically, it is always true that $W \leq C \leq M$, where

$$
W(u)=\max \left(\sum_{i=1}^{n} u_{i}-n+1,0\right) \quad \text { and } \quad M(u)=\min \left(u_{1}, \ldots, u_{n}\right) .
$$

While $M$ is a copula in any dimension, $W$ fails to be a distribution function when $n>2$. When $M$ is the underlying copula of a vector $X=\left(X_{1}, \ldots, X_{n}\right)$, the components of $X$ are said to be comonotonic. In the latter case, there is a random variable $U$ uniformly distributed on $(0,1)$ such that $X_{i}=F_{i}^{-1}(U)$ for all $i \in\{1, \ldots, n\}$. For more details on comonotonicity in actuarial science and finance, see Dhaene et al. $(2000,2002)$.

Finally, define $\Pi(u)=u_{1} \cdots u_{n}$ to be the copula associated with multivariate independence. The components of a random vector $X$ with underlying copula $C$ are said to be in positive lower orthant dependence (PLOD) if $C \geq \Pi$ and in positive upper orthant dependence (PUOD) when $C^{\mathrm{d}} \leq \Pi^{\mathrm{d}}$. If $X$ is both PLOD and PUOD, $X$ is said to be positively orthant dependent (POD). In the bivariate case, these notions are equivalent, and $X$ is said to be positive quadrant dependent (PQD). For a testing procedure that checks whether the components of a random vector are PQD, see Scaillet (2005).

\section{Bounds when the marginal distributions are known}

Consider the risks $X_{1}, \ldots, X_{n}$, i.e., $n$ non-negative random variables with known continuous distribution functions $F_{1}, \ldots, F_{n}$, respectively. It is assumed throughout this section that the copula $C$ underlying the distribution of $\left(X_{1}, \ldots, X_{n}\right)$ is unknown. It will be supposed, however, that partial information is available about $C$, namely that there are copulas $C_{\mathrm{L}}$ and $C_{\mathrm{U}}$ such that $C \geq C_{\mathrm{L}}$ and $C^{\mathrm{d}} \leq C_{\mathrm{U}}^{\mathrm{d}}$.

Now denote by $F_{S}$ the distribution function of $S=X_{1}+\cdots+X_{n}$. In order to derive stochastic bounds on the value-at-risk of $S$, an $n$-variate analogue of a result due to Makarov (1981) and independently found by Rüschendorf (1982) will be recalled. The multivariate version presented herein can be found in Cossette et al. (2002). Explicitly, 
one has $\underline{F}(s) \leq F_{S}(s) \leq \bar{F}(s)$, where

$$
\underline{F}(s)=\sup _{u_{1}+\cdots+u_{n}=s} C_{\mathrm{L}}\left\{F_{1}\left(u_{1}\right), \ldots, F_{n}\left(u_{n}\right)\right\}
$$

and

$$
\bar{F}(s)=\inf _{u_{1}+\cdots+u_{n}=s} C_{\mathrm{U}}^{\mathrm{d}}\left\{F_{1}\left(u_{1}\right), \ldots, F_{n}\left(u_{n}\right)\right\}
$$

Note in passing that $\underline{F}$ and $\bar{F}$ are themselves distribution functions. Frank et al. (1987) proved the best-possible nature of these bounds. Williamson and Downs (1990) translated these results into bounds for the value-at-risk of the sum of two risks using the duality principle. The $n$-dimensional formulation of this result is stated formally in the next theorem. This is in fact a special case of Theorem 3.1 of Embrechts et al. (2003), where the value-at-risk of a function $\psi\left(x_{1}, \ldots, x_{n}\right)$ of $n$-dependent risks was treated, applying the duality principle of Frank and Schweizer (1979). In all that follows, $\operatorname{VaR}_{\alpha}(S)$ stands for the value-at-risk, at level $\alpha$, of the sum of $n$ risks.

Theorem 3.1. Let $X_{1}, \ldots, X_{n}$ be $n$ risks with continuous marginal distribution functions $F_{1}, \ldots, F_{n}$, respectively. If the unknown copula $C$ of $\left(X_{1}, \ldots, X_{n}\right)$ is such that $C \geq C_{\mathrm{L}}$ and $C^{\mathrm{d}} \leq C_{\mathrm{U}}^{\mathrm{d}}$ for some copulas $C_{\mathrm{L}}$ and $C_{\mathrm{U}}$, then

$$
\underline{\operatorname{VaR}}_{C_{\mathrm{U}}}(\alpha) \leq \operatorname{VaR}_{\alpha}(S) \leq \overline{\operatorname{VaR}}_{C_{\mathrm{L}}}(\alpha)
$$

where

$$
\underline{\operatorname{VaR}}_{C_{\mathrm{U}}}(\alpha)=\sup _{C_{\mathrm{U}}^{\mathrm{d}}\left(u_{1}, \ldots, u_{n}\right)=\alpha} \sum_{i=1}^{n} F_{i}^{-1}\left(u_{i}\right)
$$

and

$$
\overline{\operatorname{VaR}}_{C_{\mathrm{L}}}(\alpha)=\inf _{C_{\mathrm{L}}\left(u_{1}, \ldots, u_{n}\right)=\alpha} \sum_{i=1}^{n} F_{i}^{-1}\left(u_{i}\right) .
$$

For fixed marginal distribution functions $F_{1}, \ldots, F_{n}$, the dependence scenario leading to the worst possible valueat-risk is not attained under comonotonicity of the risks. In other words, there exist dependence structures such that $\operatorname{VaR}_{\alpha}(S)$ strictly exceeds the value-at-risk of $n$ comonotonic risks, which can be seen to be

$$
F_{1}^{-1}(\alpha)+\cdots+F_{n}^{-1}(\alpha)
$$

For this reason, value-at-risk is not a coherent risk measure. The worst-case copula is rather given by

$$
C_{\alpha}(u)= \begin{cases}\max \left\{C_{\mathrm{L}}(u), \alpha\right\}, & u \in[\alpha, 1]^{n} \\ M(u), & \text { otherwise }\end{cases}
$$

This result was shown by Frank et al. (1987), and by Rüschendorf (1982) when $n=2$ and $C_{\mathrm{L}}=W$. The copulas $C_{\alpha}$ have recently been investigated by Embrechts et al. (2005), who provide many interesting graphical interpretations. 
Solutions to optimization problems like those of Eqs. (1) and (2) are commonly computed using the Lagrange multiplier. For example, the solution of (2) is obtained by solving the system:

$$
\left(F_{i}^{-1}\right)^{\prime}\left(u_{i}\right)=\lambda \frac{\partial}{\partial u_{i}} C_{\mathrm{L}}\left(u_{1}, \ldots, u_{n}\right), \quad 1 \leq i \leq n \text { and } C_{\mathrm{L}}\left(u_{1}, \ldots, u_{n}\right)=\alpha .
$$

However, an approach that will prove easier to handle in the sequel is to reformulate (1) and (2) in an optimization problem involving only $n-1$ variables. For that purpose, let $u^{\backslash n}=\left(u_{1}, \ldots, u_{n-1}\right)$ be the vector obtained by removing the $n$th component of $u=\left(u_{1}, \ldots, u_{n}\right)$. Then, for $u^{\backslash n}$ fixed, introduce the non-decreasing functions:

$$
x \mapsto C_{u^{\backslash n}}(x)=C\left(u^{\backslash n}, x\right) \quad \text { and } \quad x \mapsto C_{u^{\backslash n}}^{\mathrm{d}}(x)=C^{\mathrm{d}}\left(u^{\backslash n}, x\right)
$$

and denote by $C_{u^{\backslash n}}^{-1},\left(C_{u^{\natural n}}^{\mathrm{d}}\right)^{-1}$ their respective generalized left-continuous inverse, as described in Definition 2.1. The following straightforward adaptation of Theorem 3.1 can now be stated.

Proposition 3.1. Let $X_{1}, \ldots, X_{n}$ be $n$ risks with continuous marginal distribution functions $F_{1}, \ldots, F_{n}$, respectively. If the unknown copula $C$ of $\left(X_{1}, \ldots, X_{n}\right)$ is such that $C \geq C_{\mathrm{L}}$ and $C^{\mathrm{d}} \leq C_{\mathrm{U}}^{\mathrm{d}}$ for some copulas $C_{\mathrm{L}}$ and $C_{\mathrm{U}}$, then

$$
\underline{\operatorname{VaR}}_{C_{\mathrm{U}}}(\alpha) \leq \operatorname{VaR}_{\alpha}(S) \leq \overline{\operatorname{VaR}}_{C_{\mathrm{L}}}(\alpha),
$$

where

$$
\underline{\mathrm{VaR}}_{C_{\mathrm{U}}}(\alpha)=\sup _{C_{\mathrm{U}}^{\mathrm{d}}\left(u^{n}, 0\right) \leq \alpha}\left[\sum_{i=1}^{n-1} F_{i}^{-1}\left(u_{i}\right)+F_{n}^{-1}\left\{\left(C_{\mathrm{U}, u^{\backslash n}}^{\mathrm{d}}\right)^{-1}(\alpha)\right\}\right]
$$

and

$$
\overline{\operatorname{VaR}}_{C_{\mathrm{L}}}(\alpha)=\inf _{C_{\mathrm{L}}\left(u^{\backslash n}, 1\right) \geq \alpha}\left[\sum_{i=1}^{n-1} F_{i}^{-1}\left(u_{i}\right)+F_{n}^{-1}\left\{C_{\mathrm{L}, u \backslash^{n}}^{-1}(\alpha)\right\}\right] .
$$

In practical situations, the dependence structure of $\left(X_{1}, \ldots, X_{n}\right)$ is often unknown. However, for any copula $C$, the inequalities $C(u) \geq W(u)$ and $C^{\mathrm{d}}(u) \leq \tilde{W}^{\mathrm{d}}(u)=\min \left(1, u_{1}+\cdots+u_{n}\right)$ hold. Note that $\tilde{W}^{\mathrm{d}}=W^{\mathrm{d}}$ only for $n=2$. Hence, in view of Proposition 3.1, it is always true that

$$
\underline{\operatorname{VaR}}_{\tilde{W}}(\alpha) \leq \operatorname{VaR}_{\alpha}(S) \leq \overline{\operatorname{VaR}}_{W}(\alpha),
$$

even if $W$ is not a copula and $\tilde{W}^{\mathrm{d}}$ is not the dual of a copula when $n>2$. In fact, only the property that $C_{\mathrm{L}}$ and $C_{\mathrm{U}}^{\mathrm{d}}$ are increasing in each of their arguments was necessary to establish Theorem 3.1, and as a consequence Proposition 3.1.

These bounds can potentially be tightened whenever additional information guarantees that there exist copulas $C_{0}$ and $C_{1}$ such that $C \geq C_{0}>W$ and $C^{\mathrm{d}} \leq C_{1}^{\mathrm{d}}<\tilde{W}^{\mathrm{d}}$. Indeed, since this implies that

$$
C_{0, u^{\backslash n}}^{-1}(\alpha)<W_{u^{\backslash n}}^{-1}(\alpha) \text { and }\left(C_{1, u^{\natural n}}^{\mathrm{d}}\right)^{-1}(\alpha)>\left(\tilde{W}_{u \backslash^{\natural}}^{\mathrm{d}}\right)^{-1}(\alpha) \text {, }
$$

one concludes that

$$
\underline{\operatorname{VaR}}_{C_{1}}(\alpha) \geq \underline{\operatorname{VaR}}_{\tilde{W}}(\alpha) \text { and } \quad \overline{\operatorname{VaR}}_{C_{0}}(\alpha) \leq \overline{\operatorname{VaR}}_{W}(\alpha)
$$


from the fact that $F_{n}^{-1}$ is non-decreasing and the optimisation regions are larger. In the bivariate case, the knowledge that $C \geq C_{0}$ can lead to a simultaneous improvement of $\underline{\operatorname{VaR}}_{\tilde{W}}(\alpha)=\underline{\operatorname{VaR}}_{W}(\alpha)$ and $\overline{\operatorname{VaR}}_{W}(\alpha)$, since it implies that $C^{\mathrm{d}} \leq C_{0}^{\mathrm{d}}$.

Now in order to apply the arguments in the above discussion, assume that $X_{1}, \ldots, X_{n}$ are positively lower orthant dependent (PLOD), i.e., $C \geq \Pi$. In this case, the possibly improved upper bound is $\overline{\operatorname{VaR}}_{\Pi}(\alpha)$. In other contexts, it can be supposed that the risks are in positive upper orthant dependence (PUOD), which means that $C^{\mathrm{d}} \leq \Pi^{\mathrm{d}}$. This can lead to a better lower bound, namely $\underline{\operatorname{VaR}}_{\Pi}(\alpha)$. However, these assumptions of PLOD and PUOD risks are rather imprudent in VaR-based risk management.

Remark 3.1. Interestingly, the bounds (3) and (4) of Proposition 3.1 cannot be improved even if available information tells that $C$ is bounded above. For example, no improvement is achieved even if it is known that the copula $C$ of $\left(X_{1}, \ldots, X_{n}\right)$ satisfies $C \leq \Pi$.

Unfortunately, explicit solutions to (3) or (4) are not always available. One then has to rely on numerical solutions. However, it will be seen that easily computable expressions can arise by making assumptions about the densities of the risks, which ensure that the function to be optimized is convex.

The next proposition generalizes previous findings made by Embrechts et al. (2000), where $\overline{\operatorname{VaR}}_{W}(\alpha)$ was computed for the sum of two identically distributed Pareto and Gamma risks $X_{1}$ and $X_{2}$. The result presented here holds true whenever the common density $f$ of $n$ risks is non-increasing above a certain threshold and applies to any exchangeable copula $C_{\mathrm{L}}$ such that $C \geq C_{\mathrm{L}}$. Before stating it, assume that $f$ is differentiable.

Proposition 3.2. Let $X_{1}, \ldots, X_{n}$ be $n$ risks with common distribution function $F$ and unknown copula $C$, and suppose Assumptions A1 and A2 below hold true.

A1. There exists $x^{\star} \in \mathbb{R}$ such that $f(x)=\mathrm{d} F(x) / \mathrm{d} x$ is non-increasing for all $x \geq x^{\star}$.

A2. There is an exchangeable copula $C_{\mathrm{L}}$ such that $C \geq C_{\mathrm{L}}$ and

$$
\frac{\partial^{2}}{\partial u_{i} \partial u_{j}} C_{\mathrm{L}, u^{\natural n}}^{-1} \geq 0 \text { for any } 1 \leq i, j \leq n-1 \text {. }
$$

Then, for $\alpha \geq F\left(x^{\star}\right)$, one has that $\operatorname{VaR}_{\alpha}(S) \leq \overline{\operatorname{VaR}}_{C_{\mathrm{L}}}(\alpha)$, where

$$
\overline{\operatorname{VaR}}_{C_{\mathrm{L}}}(\alpha)=n F^{-1}\left\{\delta_{C_{\mathrm{L}}}^{-1}(\alpha)\right\},
$$

with $\delta_{C_{\mathrm{L}}}(t)=C_{\mathrm{L}}(t, \ldots, t)$ being the diagonal section of $C_{\mathrm{L}}$.

Proof. Define $s_{\alpha}\left(u^{\backslash n}\right)=C_{\mathrm{L}, u^{\natural n}}^{-1}(\alpha)$ and $G(u)=F^{-1}(u)$. From Eq. (4) of Proposition 3.1, one can write

$$
\overline{\operatorname{VaR}}_{C_{\mathrm{L}}}(\alpha)=\inf _{C_{\mathrm{L}}\left(u^{n}, 1\right) \geq \alpha} g\left(u^{\backslash n}\right),
$$

where

$$
g\left(u^{\backslash n}\right)=\sum_{i=1}^{n-1} G\left(u_{i}\right)+G \circ s_{\alpha}\left(u^{\backslash n}\right) .
$$


By Assumption A1, one deduces that $G^{\prime \prime}(u) \geq 0$ for all $u \geq F\left(x^{\star}\right)$, and in particular for $\alpha \leq u \leq 1$ since $\alpha \geq F\left(x^{\star}\right)$. Furthermore, $G^{\prime}(u)=\left\{f \circ F^{-1}(u)\right\}^{-1} \geq 0$ for all $u$. Next, note that

$$
\frac{\partial^{2}}{\partial u_{i} \partial u_{j}} g\left(u^{\backslash n}\right)= \begin{cases}h_{i j}\left(u^{\backslash n}\right)+G^{\prime \prime}\left(u_{i}\right), & i=j, \\ h_{i j}\left(u^{\backslash n}\right), & i \neq j,\end{cases}
$$

where

$$
h_{i j}\left(u^{\backslash n}\right)=G^{\prime \prime} \circ s_{\alpha}\left(u^{\backslash n}\right) s_{\alpha}^{(i)}\left(u^{\backslash n}\right) s_{\alpha}^{(j)}\left(u^{\backslash n}\right)+G^{\prime} \circ s_{\alpha}\left(u^{\backslash n}\right) s_{\alpha}^{(i j)}\left(u^{\backslash n}\right)
$$

$s_{\alpha}^{(i)}=\partial s_{\alpha} / \partial u_{i}$ and $s_{\alpha}^{(i j)}=\partial^{2} s_{\alpha} / \partial u_{i} \partial u_{j}$. By Assumption A2, $s_{\alpha}^{(i j)} \geq 0$. This, coupled with the fact that

$$
s_{\alpha}^{(i)}\left(u^{\backslash n}\right)=-\frac{C_{\mathrm{L}}^{(i)}\left\{u^{\backslash n}, s_{\alpha}\left(u^{\backslash n}\right)\right\}}{C_{\mathrm{L}}^{(n)}\left\{u^{\backslash n}, s_{\alpha}\left(u^{\backslash n}\right)\right\}} \leq 0,
$$

where $C_{\mathrm{L}}^{(i)}$ is the first-order partial derivative of $C_{\mathrm{L}}$ with respect to its $i$ th component, allows one to conclude that $g$ is convex on $[\alpha, 1]^{n-1}$. A possible minimum is then attained where the first order derivatives of $g$ vanish, i.e., at the point $\tilde{u}^{\backslash n}$ such that

$$
\frac{G^{\prime}\left(\tilde{u}_{i}\right)}{s_{\alpha}^{(i)}\left(\tilde{u}^{\backslash n}\right)}+G^{\prime} \circ s_{\alpha}\left(\tilde{u}^{\backslash n}\right)=0, \quad 1 \leq i \leq n-1 .
$$

A natural candidate is $\tilde{u}^{\backslash n}$, which satisfies $\tilde{u}_{i}=s_{\alpha}\left(\tilde{u}^{\backslash n}\right)$ for all $i \in\{1, \ldots, n-1\}$. This would imply that $\tilde{u}_{1}=$ $\cdots=\tilde{u}_{n-1}=\tilde{u}$ and henceforth $\tilde{u}$ would be such that $C_{\mathrm{L}}(\tilde{u}, \ldots, \tilde{u})=\delta_{C_{\mathrm{L}}}(\tilde{u})=\alpha$, or equivalently $\tilde{u}=\delta_{C_{\mathrm{L}}}^{-1}(\alpha)$. This solution satisfies the equations in (6) since, using the exchangeability of $C_{\mathrm{L}}$,

$$
s_{\alpha}^{(i)}\left(\tilde{u}^{\backslash n}\right)=-\frac{C_{\mathrm{L}}^{(i)}\left\{\tilde{u}^{\backslash n}, s_{\alpha}\left(\tilde{u}^{\backslash n}\right)\right\}}{C_{\mathrm{L}}^{(n)}\left\{\tilde{u}^{\backslash n}, s_{\alpha}\left(\tilde{u}^{\backslash n}\right)\right\}}=-\frac{C_{\mathrm{L}}^{(i)}(\tilde{u}, \ldots, \tilde{u})}{C_{\mathrm{L}}^{(n)}(\tilde{u}, \ldots, \tilde{u})}=-1 .
$$

Finally, this solution belongs to the optimization region since

$$
C_{\mathrm{L}}\left(\tilde{u}^{\backslash n}, 1\right) \geq C_{\mathrm{L}}\left(\tilde{u}^{\backslash n}, \tilde{u}\right)=\delta_{C_{\mathrm{L}}}(\tilde{u})=\alpha .
$$

Remark 3.2. For a distribution function $F$ whose associated density satisfies Assumption A1, one obtains easily that for all $\alpha \geq F\left(x^{\star}\right)$ :

$$
\overline{\operatorname{VaR}}_{W}(\alpha)=n F^{-1}\left(\frac{\alpha+n-1}{n}\right) \text { and } \overline{\operatorname{VaR}}_{\Pi}(\alpha)=n F^{-1}\left(\alpha^{1 / n}\right)
$$

since Assumption A2 is met for both $W$ and $\Pi$. Interestingly, it is clear that $\overline{\operatorname{VaR}}_{W}(\alpha)$ exceeds the value-at-risk of the sum of $n$ comonotonic risks, namely $n F^{-1}(\alpha)$.

Assumption A1 is fulfilled for many important models in finance. In fact, as long as A2 holds true, the conclusion of Proposition 3.2 applies for all $\alpha \geq 0$ when the density is non-increasing on its entire domain, as is the case with the exponential and Pareto models. Even for unimodal densities, the range of $\alpha$ where the result holds true can be wide enough for applications. As an illustration, let $F_{a, b}$ be the distribution function of a gamma random variable with 
Table 1

Values of $F_{a, 1}(a-1)$, where $F_{a, 1}$ is the distribution function of a $\operatorname{Gamma}(a, 1)$ distribution

\begin{tabular}{ll}
\hline$a$ & $\alpha$ \\
\hline 1 & 0.000 \\
1.5 & 0.199 \\
2 & 0.264 \\
3 & 0.323 \\
4 & 0.353 \\
5 & 0.371 \\
10 & 0.413 \\
$\infty$ & 0.500 \\
\hline
\end{tabular}

parameters $a$ and $b$. The associated density is known to be non-increasing for all $x \geq x^{\star}=(a-1) b$. Table 1 provides some values of $F_{a, b}\left(x^{\star}\right)=F_{a, 1}(a-1)$, i.e., the minimum values of $\alpha$ for which the upper bound of Proposition 3.2 is still valid.

Here, the condition $\alpha \geq F_{a, b}\left(x^{\star}\right)$ that appears in Proposition 3.2 is not restrictive in practice since one is usually interested in the computation of the value-at-risk at large values of $\alpha$. Note that in the special case of the exponential distribution, i.e., when $a=1$, there is no restriction on $\alpha$ since the associated density is non-increasing everywhere.

Assumption A2 is satisfied as well for many copulas of interest, including $W$ and $\Pi$. More generally, one can show that A2 is true for any Archimedean copula, i.e., for dependence models of the form

$$
C\left(u_{1}, \ldots, u_{n}\right)=\phi^{-1}\left\{\phi\left(u_{1}\right)+\cdots+\phi\left(u_{n}\right)\right\},
$$

where $\phi:(0,1] \rightarrow[0, \infty)$ is a bijective generator such that

$$
(-1)^{i} \frac{\mathrm{d}^{i}}{\mathrm{~d} t^{i}} \phi^{-1}(t)>0, \quad 1 \leq i \leq n \text { and } \phi(1)=0 .
$$

Many widely used multivariate families fall into this category, including the Clayton-Oakes models and the extremevalue Gumbel copulas. For more details on Archimedean copulas, see Genest and MacKay (1986) or Chapter 4 of the monograph by Nelsen (1999).

Now an explicit expression for the lower bound to be found in Theorem 3.1 will be given when no information about the dependence structure of $n$ risks is available. In that case, one deduces from Eq. (1) that $\operatorname{VaR}_{\alpha}(S)$ is bounded below by

$$
\underline{\operatorname{VaR}}_{\tilde{W}}(\alpha)=\sup _{u_{1}+\cdots+u_{n}=\alpha} \sum_{i=1}^{n} F_{i}^{-1}\left(u_{i}\right),
$$

since it is always true that $C^{\mathrm{d}} \leq \tilde{W}^{\mathrm{d}}=\min \left(1, u_{1}+\cdots+u_{n}\right)$. If the densities associated to the risks are nonincreasing on a given range, then the function to be maximized will be convex. This is a key requirement in the proof of the next result, where it is assumed that for all $i \in\{1, \ldots, n\}$, the density $f_{i}$ of $X_{i}$ is differentiable.

Proposition 3.3. Suppose that for each $i \in\{1, \ldots, n\}$, there exist a number $x_{i}^{\star}$ such that $f_{i}(x)$ is non-increasing for all $x \leq x_{i}^{\star}$. Then, for $\alpha \leq \min \left\{F_{1}\left(x_{1}^{\star}\right), \ldots, F_{n}\left(x_{n}^{\star}\right)\right\}$, one has

$$
\underline{\operatorname{VaR}}_{\tilde{W}}(\alpha)=\max _{1 \leq i \leq n}\left\{F_{i}^{-1}(\alpha)+\sum_{1 \leq j \neq i \leq n} F_{j}^{-1}(0)\right\} .
$$


Proof. The proof will proceed by induction. First note that by assumption, $-f_{i}^{\prime} \circ F_{i}^{-1}(u) \geq 0$ for all $u \leq F_{i}\left(x_{i}^{\star}\right)$, so that for all $0 \leq u \leq \alpha \leq F_{i}\left(x_{i}^{\star}\right)$ :

$$
\left(F_{i}^{-1}(u)\right)^{\prime \prime}=\frac{-f_{i}^{\prime} \circ F_{i}^{-1}(u)}{\left\{f_{i} \circ F_{i}^{-1}(u)\right\}^{3}} \geq 0, \quad 1 \leq i \leq n .
$$

For $n=2$, one deduces from Eq. (7) that

$$
\underline{\operatorname{VaR}}_{\tilde{W}}(\alpha)=\sup _{0 \leq u \leq \alpha}\left\{F_{1}^{-1}(u)+F_{2}^{-1}(\alpha-u)\right\}=\max \left\{F_{1}^{-1}(\alpha)+F_{2}^{-1}(0), F_{1}^{-1}(0)+F_{2}^{-1}(\alpha)\right\}
$$

where the convexity of $F_{1}^{-1}$ and $F_{2}^{-1}$ was used.

Now suppose Eq. (8) is true for a given $n \geq 2$ and let $u_{1}, \ldots, u_{n+1}$ be any non-negative numbers that satisfy $u_{1}+\cdots+u_{n+1}=\alpha$. By the induction hypothesis:

$$
\sum_{i=1}^{n} F_{i}^{-1}\left(u_{i}\right) \leq \max _{1 \leq i \leq n}\left\{F_{i}^{-1}\left(\alpha-u_{n+1}\right)+\sum_{1 \leq i \neq j \leq n} F_{j}^{-1}(0)\right\}
$$

because $u_{1}+\cdots+u_{n}=\alpha-u_{n+1}$. Hence,

$$
\sum_{i=1}^{n+1} F_{i}^{-1}\left(u_{i}\right) \leq \max _{1 \leq i \leq n}\left\{F_{i}^{-1}\left(\alpha-u_{n+1}\right)+F_{n+1}^{-1}\left(u_{n+1}\right)+\sum_{1 \leq j \neq i \leq n} F_{j}^{-1}(0)\right\},
$$

and since the result holds for $n=2$,

$$
F_{i}^{-1}\left(\alpha-u_{n+1}\right)+F_{n+1}^{-1}\left(u_{n+1}\right) \leq \max \left\{F_{i}^{-1}(\alpha)+F_{n+1}^{-1}(0), F_{n+1}^{-1}(\alpha)+F_{i}^{-1}(0)\right\} .
$$

It follows that

$$
\sum_{i=1}^{n+1} F_{i}^{-1}\left(u_{i}\right) \leq \max _{1 \leq i \leq n}\left\{F_{i}^{-1}(\alpha)+\sum_{1 \leq j \neq i \leq n+1} F_{j}^{-1}(0)\right\}
$$

and therefore

$$
\sup _{u_{1}+\cdots+u_{n+1}=\alpha} \sum_{i=1}^{n+1} F_{i}^{-1}\left(u_{i}\right)=\max _{1 \leq i \leq n}\left\{F_{i}^{-1}(\alpha)+\sum_{1 \leq j \neq i \leq n+1} F_{j}^{-1}(0)\right\} .
$$

Consequently, (8) is true for $n+1$.

Remark 3.3. It is not possible to obtain an analogous version of the last proposition for any copula $C_{\mathrm{U}}$ such that $C^{\mathrm{d}} \leq C_{\mathrm{U}}^{\mathrm{d}}$, since the function to be maximized will no longer be convex. However, a result similar to that of Proposition 3.2 is possible for the lower bound when it is supposed that the common density of $n$ risks is nondecreasing for all $x \leq x^{\star}$. The gamma model, among others, satisfies this requirement. Under the assumption that 
there exists an exchangeable copula $C_{\mathrm{U}}$ such that $C^{\mathrm{d}} \leq C_{\mathrm{U}}^{\mathrm{d}}$ and

$$
\frac{\partial^{2}}{\partial u_{i} \partial u_{j}}\left(C_{\mathrm{U}, u^{\backslash n}}^{\mathrm{d}}\right)^{-1} \leq 0 \text { for any } 1 \leq i, j \leq n-1,
$$

the function to be maximized in Eq. (3) of Proposition 3.1 is concave. It is hence established from this fact, using arguments identical to that of the proof of Proposition 3.2, that the maximum is attained at $\tilde{u}_{i}=\left(\delta_{C_{\mathrm{U}}}^{\mathrm{d}}\right)^{-1}(\alpha), 1 \leq$ $i \leq n$, where $\delta_{C_{\mathrm{U}}}^{\mathrm{d}}(t)=C_{\mathrm{U}}^{\mathrm{d}}(t, \ldots, t)$. As a consequence, for $\alpha \leq F\left(x^{\star}\right)$ :

$$
\underline{\mathrm{VaR}}_{C_{\mathrm{U}}}(\alpha)=n F^{-1}\left\{\delta_{C_{\mathrm{U}}^{-1}}^{\mathrm{d}}(\alpha)\right\}
$$

This result is of limited application, however, since it holds true only for small values of $\alpha$.

As an illustration of the latter remark, let $F$ by a distribution function whose associated density $f(x)$ is nondecreasing for all $x \leq x^{\star}$. One can see that for all $\alpha \leq F\left(x^{\star}\right)$ :

$$
\underline{\operatorname{VaR}}_{\tilde{W}}(\alpha)=n F^{-1}\left(\frac{\alpha}{n}\right) \quad \text { and } \quad \underline{\operatorname{VaR}}_{\Pi}(\alpha)=n F^{-1}\left\{1-(1-\alpha)^{1 / n}\right\}
$$

\section{Bounds when the marginal distributions are unknown}

In this section, lower and upper bounds for $\operatorname{VaR}_{\alpha}(S)$ are proposed for cases where only the first two moments of $X_{1}, \ldots, X_{n}$ are known. The main result will make use of the univariate extremal distributions given by Kaas and Goovaerts (1986) when only the first two moments of a random variable are known. Specifically, let $X$ be a random variable with unknown distribution function $F$ and known moments $\mathrm{E}(X)=\mu_{X}>0$ and $\operatorname{var}(X)=\sigma_{X}^{2}>0$. These authors showed that $\underline{F}_{\mu_{X}, \sigma_{X}}(x) \leq F(x) \leq \bar{F}_{\mu_{X}, \sigma_{X}}(x)$, where

$$
\underline{F}_{\mu_{X}, \sigma_{X}}(x)= \begin{cases}0, & 0 \leq x \leq \mu_{X}, \\ \frac{x-\mu_{X}}{x}, & \mu_{X}<x \leq \frac{\sigma_{X}^{2}+\mu_{X}^{2}}{\mu_{X}}, \\ \frac{\left(x-\mu_{X}\right)^{2}}{\left(x-\mu_{X}\right)^{2}+\sigma_{X}^{2}}, & x>\frac{\sigma_{X}^{2}+\mu_{X}^{2}}{\mu_{X}}\end{cases}
$$

and

$$
\bar{F}_{\mu_{X}, \sigma_{X}}(x)= \begin{cases}\frac{\sigma_{X}^{2}}{\sigma_{X}^{2}+\left(x-\mu_{X}\right)^{2}}, & 0 \leq x \leq \mu_{X}, \\ 1, & x>\mu_{X} .\end{cases}
$$

The following proposition translates these bounds in terms of the value-at-risk of a single random variable $X$. To achieve this, it suffices to invert the previous extremal distributions. Before stating it, define on $[0,1]$ the strictly increasing function $q(u)=\sqrt{u /(1-u)}$ and let

$$
g_{a, b}(u)=\{a-b q(1-u)\} \mathbf{1}\left(u \geq \frac{b^{2}}{a^{2}+b^{2}}\right)
$$


and

$$
h_{a, b}(u)=a+a q^{2}(u) \mathbf{1}\left(u \leq \frac{b^{2}}{a^{2}+b^{2}}\right)+b q(u) \mathbf{1}\left(u>\frac{b^{2}}{a^{2}+b^{2}}\right),
$$

where $\mathbf{1}(\cdot)$ stands for the indicator function of a set.

Proposition 4.1. If $X$ is a random variable with mean $\mu_{X}$ and variance $\sigma_{X}^{2}$,

$$
\underline{\operatorname{VaR}}_{\mu_{X}, \sigma_{X}}(\alpha) \leq \operatorname{VaR}_{\alpha}(X) \leq \overline{\operatorname{VaR}}_{\mu_{X}, \sigma_{X}}(\alpha),
$$

where

$$
\underline{\operatorname{VaR}}_{\mu_{X}, \sigma_{X}}(\alpha)=\bar{F}_{\mu_{X}, \sigma_{X}}^{-1}(\alpha)=g_{\mu_{X}, \sigma_{X}}(\alpha)
$$

and

$$
\overline{\operatorname{VaR}}_{\mu_{X}, \sigma_{X}}(\alpha)=\underline{F}_{\mu_{X}, \sigma_{X}}^{-1}(\alpha)=h_{\mu_{X}, \sigma_{X}}(\alpha) .
$$

Now in order to derive bounds for the value-at-risk of $S=X_{1}+\cdots+X_{n}$, an approach will be used which is similar to that explored by Genest et al. (2002) in a stop-loss premium context. Specifically, let $X_{1}, \ldots, X_{n}$ be $n$ risks such that $\mathrm{E}\left(X_{i}\right)=\mu_{i}>0$ and $\operatorname{var}\left(X_{i}\right)=\sigma_{i}^{2}>0$ are known. Hence, if $R$ stands for their associated correlation matrix, the first two moments of the single random variable $S$ are expressed as

$$
\mu=\mathrm{E}(S)=\mu_{1}+\cdots+\mu_{n} \quad \text { and } \quad \sigma^{2}(R)=\operatorname{var}(S)=\sum_{i=1}^{n} \sigma_{i}^{2}+2 \sum_{i<j} \sigma_{i} \sigma_{j} R_{i j} .
$$

From Proposition 4.1, possible bounds for $\operatorname{VaR}_{\alpha}(S)$ are then

$$
\underline{\operatorname{VaR}}_{\mu, \sigma(R)}(\alpha) \leq \operatorname{VaR}_{\alpha}(S) \leq \overline{\operatorname{VaR}}_{\mu, \sigma(R)}(\alpha) .
$$

Since these two extremal value-at-risk depend on the unknown elements of $R$, expressions for the lower and the upper bounds that are free of $R$ are obtained by minimizing $\underline{\operatorname{VaR}}_{\mu, \sigma(R)}$ and maximizing $\overline{\operatorname{VaR}}_{\mu, \sigma(R)}$ with respect to $R_{i j} \in[-1,1], 1 \leq i \neq j \leq n$. These optimization problems are easily handled using the fact that $\sigma^{2}(R)$ and $t(R)=$ $\sigma^{2}(R) /\left(\sigma^{2}(R)+\mu^{2}\right)$ are strictly increasing on each of their arguments. It follows that $\underline{\operatorname{VaR}}_{\mu, \sigma(R)}$ and $\overline{\operatorname{VaR}}_{\mu, \sigma(R)}$ are respectively decreasing and increasing functions of $R_{i j}$, in the strict sense, so that both solutions are achieved when $R_{i j}=1$ for all $i, j \in\{1, \ldots, n\}$. As a consequence, the resulting bounds depend only on $\mu=\mu_{1}+\cdots+\mu_{n}$ and $\sigma=\sigma_{1}+\cdots+\sigma_{n}$. These new findings are summarized in the proposition below.

Proposition 4.2. Let $X_{1}, \ldots, X_{n}$ be $n$ risks with means $\mu_{1}, \ldots, \mu_{n}$ and variances $\sigma_{1}^{2}, \ldots, \sigma_{n}^{2}$, respectively. Then, $\underline{\operatorname{VaR}}_{\mu, \sigma}(\alpha) \leq \operatorname{VaR}_{\alpha}(S) \leq \overline{\operatorname{VaR}}_{\mu, \sigma}(\alpha)$, where

$$
\underline{\operatorname{VaR}}_{\mu, \sigma}(\alpha)=g_{\mu, \sigma}(\alpha) \text { and } \overline{\operatorname{VaR}}_{\mu, \sigma}(\alpha)=h_{\mu, \sigma}(\alpha),
$$

with $\mu=\mu_{1}+\cdots+\mu_{n}$ and $\sigma=\sigma_{1}+\cdots+\sigma_{n}$.

Remark 4.1. Since the optimization consists in taking the maximum values of $R_{i j}$ in the interval [-1, 1], the bounds in Proposition 4.2 can be improved whenever additional information guarantees that $R_{i j} \leq R_{i j}^{\star}$ for some pair $(i, j)$ 
such that $-1 \leq R_{i j}^{\star}<1$, using the fact that $\sigma(R) \leq \sigma\left(R^{\star}\right)$. One need only replace $\sigma$ by $\sigma\left(R^{\star}\right)$ in order to improve the bounds in (10).

Remark 4.2. An alternative method consists in bounding $\operatorname{VaR}_{\alpha}(S)$ via Proposition 3.1 by replacing $F_{i}^{-1}, 1 \leq i \leq n$, by $\underline{\mathrm{VaR}}_{\mu_{i}, \sigma_{i}}$ in Eq. (3) and by $\overline{\mathrm{VaR}}_{\mu_{i}, \sigma_{i}}$ in Eq. (4). If the unknown copula $C$ of $\left(X_{1}, \ldots, X_{n}\right)$ is such that $C \geq C_{\mathrm{L}}$ and $C^{\mathrm{d}} \leq C_{\mathrm{U}}^{\mathrm{d}}$ for some copulas $C_{\mathrm{L}}$ and $C_{\mathrm{U}}$, then

$$
\underline{\operatorname{VaR}}_{C_{U}}^{\star}(\alpha) \leq \operatorname{VaR}_{\alpha}(S) \leq \overline{\operatorname{VaR}}_{C_{\mathrm{L}}}^{\star}(\alpha),
$$

where

$$
\underline{\operatorname{VaR}}_{C_{\mathrm{U}}}^{\star}(\alpha)=\sup _{C_{\mathrm{U}}^{\mathrm{d}}\left(u^{\backslash n}, 0\right) \leq \alpha}\left[\sum_{i=1}^{n-1} g_{\mu_{i}, \sigma_{i}}\left(u_{i}\right)+g_{\mu_{n}, \sigma_{n}}\left\{\left(C_{\mathrm{L}, u^{\natural n}}^{\mathrm{d}}\right)^{-1}(\alpha)\right\}\right]
$$

and

$$
\overline{\operatorname{VaR}}_{C_{\mathrm{L}}}^{\star}(\alpha)=\inf _{C_{\mathrm{L}}\left(u^{\backslash n}, 1\right) \geq \alpha}\left[\sum_{i=1}^{n-1} h_{\mu_{i}, \sigma_{i}}\left(u_{i}\right)+h_{\mu_{n}, \sigma_{n}}\left\{C_{\mathrm{L}, u^{\natural n}}^{-1}(\alpha)\right\}\right] .
$$

The next proposition states that the upper bound constructed from the correlation-based methodology, namely $\overline{\mathrm{VaR}}_{\mu, \sigma}$, is uniformly better than the upper bound that arises from the copula-based approach described in the remark above, at least when the coefficients of variation of two risks $X_{1}$ and $X_{2}$ are equal. An exhaustive numerical investigation suggests that Proposition 4.3 could probably be extended to the general case. Before stating it, put

$$
t_{i}=\frac{\sigma_{i}^{2}}{\mu_{i}^{2}+\sigma_{i}^{2}}, i=1,2 \quad \text { and } \quad t=\frac{\left(\sigma_{1}+\sigma_{2}\right)^{2}}{\left(\mu_{1}+\mu_{2}\right)^{2}+\left(\sigma_{1}+\sigma_{2}\right)^{2}} .
$$

Proposition 4.3. Let $X_{1}, X_{2}$ be two risks with $\mu_{1}, \mu_{2}, \sigma_{1}, \sigma_{2}$ known and such that $\mu_{1} / \sigma_{1}=\mu_{2} / \sigma_{2}$. If $C_{\mathrm{L}}$ is any symmetric copula such that $C \geq C_{\mathrm{L}}$, then for all $0 \leq \alpha \leq 1$,

$$
\overline{\operatorname{VaR}}_{\mu, \sigma}(\alpha) \leq \overline{\operatorname{VaR}}_{C_{\mathrm{L}}}^{\star}(\alpha)
$$

Proof. By the assumption $\mu_{1} / \sigma_{1}=\mu_{2} / \sigma_{2}$, one has that $t_{1}=t_{2}=t$. Furthermore, since $C_{\mathrm{L}, u}^{-1}$ is a non-increasing function of $u$, one has that $C_{\mathrm{L}, u}^{-1}(\alpha) \geq C_{\mathrm{L}, 1}^{-1}(\alpha)=\alpha$ for any $0 \leq u \leq 1$. If $\alpha \leq t$,

$$
\begin{aligned}
\overline{\operatorname{VaR}}_{C_{\mathrm{L}}}^{\star}(\alpha) & =\mu+\min \left[\inf _{\alpha \leq u \leq t}\left\{\mu_{1} q^{2}(u)+\mu_{2} q^{2}\left(C_{\mathrm{L}, u}^{-1}(\alpha)\right)\right\}, \inf _{t<u \leq 1}\left\{\sigma_{1} q(u)+\sigma_{2} q\left(C_{\mathrm{L}, u}^{-1}(\alpha)\right)\right\}\right] \\
& \geq \mu+\min \left[\mu_{1} q^{2}(\alpha)+\mu_{2} q^{2}(\alpha), \sigma_{1} q(\alpha)+\sigma_{2} q(\alpha)\right] \\
& =\mu+\mu_{1} q^{2}(\alpha)+\mu_{2} q^{2}(\alpha)=\mu+\mu q^{2}(\alpha)=\overline{\operatorname{VaR}}_{\mu, \sigma}(\alpha),
\end{aligned}
$$

since $\sigma_{i} q(\alpha)=\mu_{i} q(t) q(\alpha) \geq \mu_{i} q^{2}(\alpha)$, while if $t<\alpha \leq 1$, one has that

$$
\overline{\operatorname{VaR}}_{C_{\mathrm{L}}}^{\star}(\alpha)=\mu+\inf _{\alpha \leq u \leq 1}\left\{\sigma_{1} q(u)+\sigma_{2} q\left(C_{\mathrm{L}, u}^{-1}(\alpha)\right)\right\} \geq \mu+\sigma_{1} q(\alpha)+\sigma_{2} q(\alpha)=\mu+\sigma q(\alpha)=\overline{\operatorname{VaR}}_{\mu, \sigma}(\alpha) .
$$

For the lower bounds derived from the correlation-based and the copula methodologies, no clear answer exists as to which approach is preferable. Indeed, it will be seen that when $n=2$, the lower bound that arises from the 
copula-based approach is the best for $0 \leq \alpha \leq t_{2}$, while the one that stems from the correlation approach is better for $t_{2}<\alpha \leq 1$. This suggests an improved lower bound for the value-at-risk for the sum of two risks by combining $\underline{\mathrm{VaR}}_{\mu, \sigma}$ and $\underline{\mathrm{VaR}}_{C \mathrm{~L}}^{\star}$. This is the object of the next proposition.

Proposition 4.4. If the first two moments $\mu_{1}, \mu_{2}, \sigma_{1}, \sigma_{2}$ of $X_{1}, X_{2}$ are known, and if $C_{\mathrm{U}}$ is a symmetric copula such that $C^{\mathrm{d}} \leq C_{\mathrm{U}}^{\mathrm{d}}$, a lower bound for $\operatorname{VaR}_{\alpha}(S)$ that is better than $\underline{\operatorname{VaR}}_{\mu, \sigma}(\alpha)$ and $\underline{\operatorname{VaR}}_{C_{\mathrm{U}}}^{\star}(\alpha)$ and that does not depend on $C_{\mathrm{U}}$ is

$$
\underline{\operatorname{VaR}}_{\mu_{1}, \mu_{2}, \sigma_{1}, \sigma_{2}}^{\star \star}(\alpha)=g_{\mu_{1}, \sigma_{1}}(\alpha)+g_{\mu_{2}, \sigma_{2}}(\alpha) .
$$

Proof. Assume without any loss of generality that $t_{1} \leq t \leq t_{2}$ and note that $\mu_{i}-\sigma_{i} q(1-\alpha) \geq 0$ if and only if $\alpha \geq t_{i}$. It will be shown that $\underline{\operatorname{VaR}}_{C_{\mathrm{U}}}^{\star}(\alpha) \geq \underline{\operatorname{VaR}}_{\mu, \sigma}(\alpha)$ for $\alpha \leq t_{2}$ and $\underline{\operatorname{VaR}}_{\mu, \sigma}(\alpha) \leq \underline{\operatorname{VaR}}_{C_{\mathrm{U}}}^{\star}(\alpha)$ for $\alpha>t_{2}$.

For $\alpha \leq t_{2}$, it is easily established that

$$
g_{\mu_{1}, \sigma_{1}}(\alpha)=g_{\mu, \sigma}(\alpha)+\left\{\mu_{1}-\sigma_{1} q(1-\alpha)\right\} \mathbf{1}\left(t_{1} \leq \alpha \leq t_{2}\right) \geq g_{\mu, \sigma}(\alpha) .
$$

Hence, since $\left(C_{\mathrm{U}, u}^{\mathrm{d}}\right)^{-1}$ is decreasing as a function of $u$, one has for all $0 \leq u \leq \alpha$ that $\left(C_{\mathrm{U}, u}^{\mathrm{d}}\right)^{-1}(\alpha) \leq\left(C_{\mathrm{U}, 0}^{\mathrm{d}}\right)^{-1}(\alpha)=$ $\alpha \leq t_{2}$, so that

$$
\begin{aligned}
\underline{\operatorname{VaR}}_{C_{\mathrm{U}}}^{\star}(\alpha) & =\sup _{0 \leq u \leq \alpha}\left[g_{\mu_{1}, \sigma_{1}}(u)+g_{\mu_{2}, \sigma_{2}}\left\{\left(C_{\mathrm{U}, u}^{\mathrm{d}}\right)^{-1}(\alpha)\right\}\right] \\
& =\sup _{0 \leq u \leq \alpha} g_{\mu_{1}, \sigma_{1}}(u)+0=g_{\mu_{1}, \sigma_{1}}(\alpha) \geq g_{\mu, \sigma}(\alpha)=\underline{\operatorname{VaR}}_{\mu, \sigma}(\alpha) .
\end{aligned}
$$

Next, when $\alpha>t_{2}$, one has that $\underline{\operatorname{VaR}}_{\mu, \sigma}(\alpha)=g_{\mu_{1}, \sigma_{1}}(\alpha)+g_{\mu_{2}, \sigma_{2}}(\alpha)$, and then

$$
\begin{aligned}
\underline{\operatorname{VaR}}_{C_{\mathrm{U}}}^{\star}(\alpha) & =\sup _{0 \leq u \leq \alpha}\left[g_{\mu_{1}, \sigma_{1}}(u)+g_{\mu_{2}, \sigma_{2}}\left\{\left(C_{\mathrm{U}, u}^{\mathrm{d}}\right)^{-1}(\alpha)\right\}\right] \leq \sup _{0 \leq u \leq \alpha} g_{\mu_{1}, \sigma_{1}}(u)+\sup _{0 \leq u \leq \alpha} g_{\mu_{2}, \sigma_{2}}\left\{\left(C_{\mathrm{U}, u}^{\mathrm{d}}\right)^{-1}(\alpha)\right\} \\
& =g_{\mu_{1}, \sigma_{1}}(\alpha)+g_{\mu_{2}, \sigma_{2}}(\alpha)=\underline{\operatorname{VaR}}_{\mu, \sigma}(\alpha) .
\end{aligned}
$$

As a consequence, a better bound is given by

$$
\underline{\operatorname{VaR}}_{\mu_{1}, \mu_{2}, \sigma_{1}, \sigma_{2}}^{\star \star}(\alpha)=g_{\mu_{1}, \sigma_{1}}(\alpha) \mathbf{1}\left(\alpha \leq t_{2}\right)+\left\{g_{\mu_{1}, \sigma_{1}}(\alpha)+g_{\mu_{2}, \sigma_{2}}(\alpha)\right\} \mathbf{1}\left(\alpha>t_{2}\right)=g_{\mu_{1}, \sigma_{1}}(\alpha)+g_{\mu_{2}, \sigma_{2}}(\alpha) .
$$

\section{Examples}

In this section, some of the bounds established in Sections 3 and 4 will be computed in the special cases when the risks are distributed as exponential and Pareto random variables. Since the densities associated to these laws are non-increasing everywhere on their domain, the results of Propositions 3.2 and 3.3 will be valid for all $0 \leq \alpha \leq 1$. The computations herein can be seen as extending Examples 1-2 in Denuit et al. (1999) in a value-at-risk context.

\subsection{Exponential risks}

Suppose $X_{1}, \ldots, X_{n}$ are distributed as shifted exponential variables, so that

$$
F_{i}^{-1}(u)=\xi_{i}-\theta_{i} \log (1-u), \quad \theta_{i}>0, \xi_{i} \geq 0
$$


When no information is available about the dependence structure of $\left(X_{1}, \ldots, X_{n}\right)$, one deduces from Theorem 3.1 that

$$
\operatorname{VaR}_{\alpha}(S) \leq \overline{\operatorname{VaR}}_{W}(\alpha)=\sum_{i=1}^{n} \xi_{i}+\inf _{u_{1}+\cdots+u_{n}=\alpha+n-1} \sum_{i=1}^{n}-\theta_{i} \log \left(1-u_{i}\right) .
$$

Since the function to be minimized is convex, the problem can be solved using the Lagrange multiplier method, which gives

$$
\frac{\theta_{i}}{1-u_{i}}=\lambda, \quad 1 \leq i \leq n, \text { and } \sum_{i=1}^{n} u_{i}=\alpha+n-1 .
$$

The solution to this system of equations is $u_{i}=1-(1-\alpha) \theta_{i}\left(\theta_{1}+\cdots+\theta_{n}\right)^{-1}$, so that

$$
\overline{\operatorname{VaR}}_{W}(\alpha)=\sum_{i=1}^{n}\left[\xi_{i}-\theta_{i} \log \left\{(1-\alpha) \frac{\theta_{i}}{\theta_{1}+\cdots+\theta_{n}}\right\}\right] .
$$

When $F_{1}=\cdots=F_{n}=F$, that is for $\xi_{1}=\cdots=\xi_{n}=\xi$ and $\theta_{1}=\cdots=\theta_{n}=\theta$, the formula above reduces to

$$
\overline{\operatorname{VaR}}_{W}(\alpha)=n \xi-n \theta \log \left(1-\frac{\alpha+n-1}{n}\right)=n F^{-1}\left(\frac{\alpha+n-1}{n}\right),
$$

as can be deduced from Proposition 3.2.

This upper bound can potentially be improved when it is known that $X_{1}, \ldots, X_{n}$ are in positive lower orthant dependence (PLOD). While no simple solution seems possible for $n>2$, one can conclude from Eq. (4) of Proposition 3.1 that an upper bound for the value-at-risk of the sum of two exponential risks is

$$
\overline{\operatorname{VaR}}_{\Pi}(\alpha)=\xi_{1}+\xi_{2}+\inf _{\alpha \leq u \leq 1} s(u), \quad \text { where } s(u)=-\theta_{1} \log (1-u)-\theta_{2} \log \left(1-\frac{\alpha}{u}\right) .
$$

From the fact that $\theta_{1}>0, \theta_{2}>0, \alpha \geq 0$ and $u \geq \alpha / 2$, it follows that $s$ is a convex function since

$$
s^{\prime \prime}(u)=\frac{\theta_{1}}{(1-u)^{2}}+\frac{\theta_{2} \alpha}{u^{2}(u-\alpha)^{2}}(2 u-\alpha) \geq 0 .
$$

Therefore, a possible minimum for $s$ is attained for $u^{\star}$ such that $s^{\prime}\left(u^{\star}\right)=0$. A straightforward computation gives the unique solution:

$$
u^{\star}=\frac{\alpha\left(\theta_{1}-\theta_{2}\right)+\sqrt{\alpha^{2}\left(\theta_{1}-\theta_{2}\right)^{2}+4 \alpha \theta_{1} \theta_{2}}}{2 \theta_{1}} .
$$

For equal distributions, it is easily seen that $u^{\star}=\sqrt{\alpha}=\delta_{\Pi}^{-1}(\alpha)$, in accordance with Proposition 3.2.

Finally, one deduces from Proposition 3.3 that

$$
\operatorname{VaR}_{\alpha}(S) \geq \underline{\operatorname{VaR}}_{\tilde{W}}(\alpha)=\sum_{i=1}^{n} \xi_{i}-\log (1-\alpha) \underset{1 \leq i \leq n}{\max } \theta_{i} .
$$

Unfortunately, no simple solution seems available when additional information ensures, e.g., that $C^{\mathrm{d}} \leq \Pi^{\mathrm{d}}$. 
Now suppose only that the first two moments of $X_{1}, \ldots, X_{n}$ are known. For the present context of exponential risks, one has

$$
\mu_{i}=\theta_{i}+\xi_{i} \quad \text { and } \quad \sigma_{i}=\theta_{i}, \quad 1 \leq i \leq n
$$

Lower and upper bounds derived from Proposition 4.2 are then given by

$$
\underline{\operatorname{VaR}}_{\xi, \theta}(\alpha)=g_{\theta+\xi, \theta}(\alpha) \quad \text { and } \quad \overline{\operatorname{VaR}}_{\xi, \theta}(\alpha)=h_{\theta+\xi, \theta}(\alpha),
$$

where $\theta=\theta_{1}+\cdots+\theta_{n}$ and $\xi=\xi_{1}+\cdots+\xi_{n}$. In the special case of two exponential risks, Proposition 4.4 gives the improved lower bound:

$$
\mathrm{VaR}_{\xi_{1}, \xi_{2}, \theta_{1}, \theta_{2}}^{\star \star}(\alpha)=g_{\theta_{1}+\xi_{1}, \theta_{1}}(\alpha)+g_{\theta_{2}+\xi_{2}, \theta_{2}}(\alpha) .
$$

In Fig. 1, the curves defined by $\underline{\operatorname{VaR}}_{\xi_{1}, \xi_{2}, \theta_{1}, \theta_{2}}^{\star V_{a R}} \underline{W}_{\tilde{W}}, \overline{\operatorname{VaR}}_{W}$ and $\overline{\mathrm{VaR}}_{\xi, \lambda}$ are displayed for the case of two exponential risks with parameter values $\xi_{1}=\xi_{2}=0$ and $\theta_{1}=\theta_{2}=1$. These bounds are compared to the value-atrisk of $S=X_{1}+X_{2}$ when the risks are supposed to be comonotonic. Table 2 reports some numerical values for popular levels of $\alpha$. As expected, the bounds for the case of known marginal distributions are much closer to the exact value-at-risk compared to the bounds when only the first two moments are known. Moreover, for large values of $\alpha$, the upper bound $\overline{\operatorname{VaR}}_{W}(\alpha)$ gives a rather good approximation to $\operatorname{VaR}_{\alpha}(S)$ compared to the performance of $\mathrm{VaR}_{\tilde{W}}(\alpha)$. In fact, one has that

$$
\lim _{\alpha \rightarrow 1} \frac{\overline{\operatorname{VaR}}_{W}(\alpha)}{\operatorname{VaR}_{\alpha}(S)}=1 \quad \text { and } \quad \lim _{\alpha \rightarrow 1} \frac{\operatorname{VaR}_{\tilde{W}}(\alpha)}{\operatorname{VaR}_{\alpha}(S)}=1 / 2 .
$$

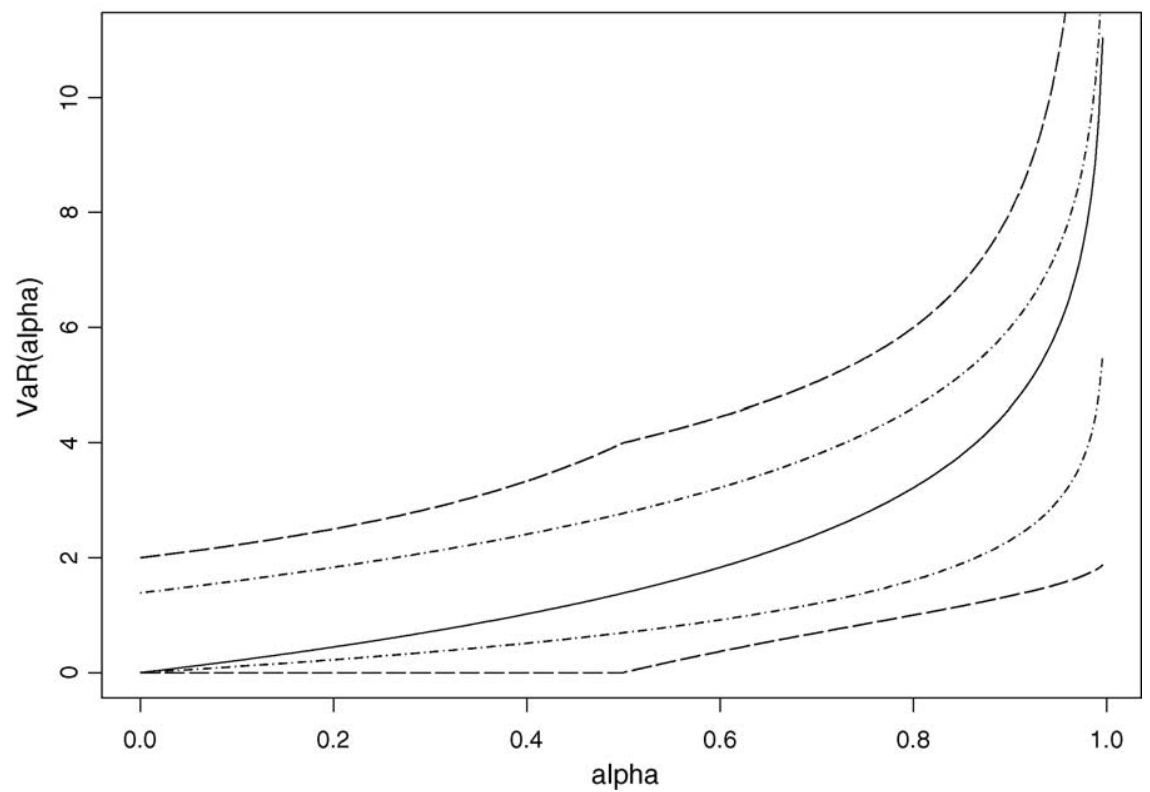

Fig. 1. Bounds on the value-at-risk of $S=X_{1}+X_{2}$ when only the first two moments are known (broken lines) and when the marginal distributions are known (dots), compared to the exact value-at-risk of $S$ (solid lines) when $X_{1}$ and $X_{2}$ are comonotonic. $X_{1}$ and $X_{2}$ are exponential with parameters $\xi_{1}=\xi_{2}=0$ and $\theta_{1}=\theta_{2}=1$. 
Table 2

Values of $\underline{\operatorname{VaR}}_{\xi}^{\star \star}, \xi_{2}, \theta_{1}, \theta_{2}, \underline{\operatorname{VaR}}_{\tilde{W}}, \operatorname{VaR}_{\alpha}, \overline{\operatorname{VaR}}_{W}$ and $\overline{\operatorname{VaR}}_{\xi, \lambda}$ for selected levels of $\alpha$

\begin{tabular}{|c|c|c|c|c|c|}
\hline$\alpha$ & $\underline{\operatorname{VaR}}_{\xi_{1}, \xi_{2}, \theta_{1}, \theta_{2}}^{\star \star}(\alpha)$ & $\underline{\operatorname{VaR}}_{W}(\alpha)$ & $\operatorname{VaR}_{\alpha}(S)$ & $\overline{\mathrm{VaR}}_{W}(\alpha)$ & $\overline{\operatorname{VaR}}_{\mu, \sigma}(\alpha)$ \\
\hline 0.900 & 1.33 & 2.30 & 4.61 & 5.99 & 8.00 \\
\hline 0.950 & 1.54 & 3.00 & 5.99 & 7.38 & 10.72 \\
\hline 0.975 & 1.68 & 3.69 & 7.38 & 8.76 & 14.49 \\
\hline 0.990 & 1.80 & 4.61 & 9.21 & 10.60 & 21.90 \\
\hline 0.995 & 1.86 & 5.30 & 10.60 & 11.98 & 30.21 \\
\hline
\end{tabular}

\subsection{Pareto risks}

Consider two risks $X_{1}$ and $X_{2}$ distributed as Pareto random variables with parameters $\gamma_{i}>0$ and $\beta_{i}>2$. In that case:

$$
F_{i}^{-1}(u)=\gamma_{i}\left\{(1-u)^{-1 / \beta_{i}}-1\right\} .
$$

From Eq. (4) of Proposition 3.1, one observes that $\overline{\operatorname{VaR}}_{W}(\alpha)=\inf _{\alpha \leq u \leq 1} h(u)$, where

$$
h(u)=\gamma_{1}\left\{(1-u)^{-1 / \beta_{1}}-1\right\}+\gamma_{2}\left\{(u-\alpha)^{-1 / \beta_{2}}-1\right\} .
$$

Since $h^{\prime \prime}(u) \geq 0$ for all $u \in[\alpha, 1]$, a possible minimum value is the real number $u^{\star}$ which solves $h^{\prime}\left(u^{\star}\right)=0$, or equivalently

$$
\frac{\gamma_{1}}{\beta_{1}}\left(1-u^{\star}\right)^{-1-1 / \beta_{1}}=\frac{\gamma_{2}}{\beta_{2}}\left(u^{\star}-\alpha\right)^{-1-1 / \beta_{2}} .
$$

A numerical routine is needed to solve this problem in general. However, an explicit solution arises when $\beta_{1}=$ $\beta_{2}=\beta$. In that case, $u^{\star}=(\alpha \kappa+1) /(\kappa+1)$, where $\kappa=\left(\gamma_{1} / \gamma_{2}\right)^{\beta /(\beta+1)}$, so that

$$
\overline{\operatorname{VaR}}_{W}(\alpha)=\left(\frac{\kappa+1}{1-\alpha}\right)^{1 / \beta}\left(\frac{\gamma_{1}}{\kappa^{1 / \beta}}+\gamma_{2}\right)-\gamma_{1}-\gamma_{2} .
$$

An improved upper bound when $\left(X_{1}, X_{2}\right)$ are known to be PQD can be computed from Eq. (4). If $\beta_{1}=\beta_{2}=\beta$, it can be shown that

$$
\overline{\operatorname{VaR}}_{\Pi}(\alpha)=\left(\gamma_{1}+\kappa^{1 / \beta} \gamma_{2}\right)\left\{\left(1-u^{\star}\right)^{-1 / \beta}-1\right\},
$$

where

$$
u^{\star}=\frac{1-\kappa+\sqrt{(1-\kappa)^{2}+4 \alpha \kappa}}{2} .
$$

For equal distributions, in which case $\kappa=1$, the above formulae reduce to

$$
\overline{\operatorname{VaR}}_{W}(\alpha)=2 \gamma\left\{\left(1-\frac{\alpha+1}{2}\right)^{-1 / \beta}-1\right\}=2 F^{-1}\left(\frac{\alpha+1}{2}\right)
$$

and

$$
\overline{\operatorname{VaR}}_{\Pi}(\alpha)=2 \gamma\left\{(1-\sqrt{\alpha})^{-1 / \beta}-1\right\}=2 F^{-1}(\sqrt{\alpha}),
$$

in accordance with the conclusion of Proposition 3.2. 
For the lower bound, one has from (8) that

$$
\underline{\operatorname{VaR}}_{\tilde{W}}(\alpha)=\max \left[\gamma_{1}\left\{(1-\alpha)^{-1 / \beta_{1}}-1\right\}, \gamma_{2}\left\{(1-\alpha)^{-1 / \beta_{2}}-1\right\}\right] .
$$

Finally, lower and upper bounds for the value-at-risk of $S=X_{1}+X_{2}$ are obtained from Propositions 4.2 and 4.4 when the only available information is about the first two moments of $X_{i}$, namely

$$
\mu_{i}=\frac{\gamma_{i}}{\beta_{i}-1} \quad \text { and } \quad \sigma_{i}^{2}=\frac{\gamma_{i}^{2} \beta_{i}}{\left(\beta_{i}-1\right)^{2}\left(\beta_{i}-2\right)}, \quad i=1,2 .
$$

\section{Acknowledgements}

The authors wish to thank Professor Christian Genest for very helpful discussions, and two referees for their meaningful comments. Research funds in partial support of this work were provided by the Natural Sciences and Engineering Research Council of Canada.

\section{References}

Artzner, P., Delbaen, F., Eber, J.-M., Heath, D., 1999. Coherent measures of risk. Mathematical Finance 9, $203-228$.

Cossette, H., Denuit, M., Marceau, É., 2002. Distributional bounds for functions of dependent risks. Mitteilungen der Schweizerische Aktuarvereinigung 45-65.

Denuit, M., Genest, C., Marceau, É., 1999. Stochastic bounds on sums of dependent risks. Insurance: Mathematics \& Economics $25,85-104$.

Dhaene, J., Denuit, M., Goovaerts, M.J., Kaas, R., Vyncke, D., 2002. The concept of comonotonicity in actuarial science and finance: theory. Insurance: Mathematics \& Economics 31, 3-33.

Dhaene, J., Wang, S., Young, V., Goovaerts, M.J., 2000. Comonotonicity and maximal stop-loss premiums. Mitteilungen der Schweizerische Aktuarvereinigung 99-113.

Embrechts, P., Höing, A., Juri, A., 2003. Using copulae to bound the value-at-risk for functions of dependent risks. Finance and Stochastics 7, $145-167$.

Embrechts, P., Höing, A., Puccetti, A., 2005. Worst VaR scenarios. Insurance: Mathematics \& Economics 37 (1), $115-134$.

Embrechts, P., McNeil, A., Straumann, D., Dempster, M., Moffat, M. (Eds.), 2000. Correlation and dependency in risk management: properties and pitfalls, Risk Management: Value at Risk and Beyond. Cambridge University Press, Cambridge, UK, pp. 176-223.

Frank, M.J., Nelsen, R., Schweizer, B., 1987. Best-possible bounds on the distribution of a sum—a problem of Kolmogorov. Probability Theory and Related Fields 7, 199-211.

Frank, M.J., Schweizer, B., 1979. On the duality of generalized infimal and supremal convolutions. Rendiconti di Matematica, Serie VI 12 (1), $1-23$.

Genest, C., Marceau, É., Mesfioui, M., 2002. Upper stop-loss bounds for sums of possibly dependent risks with given means and variances. Statistics and Probability Letters 57, 33-41.

Genest, C., MacKay, R.J., 1986. Copules archimédiennes et familles de lois bidimensionnelles dont les marges sont données. The Canadian Journal of Statistics 14, 145-159.

Kaas, R., Goovaerts, M.J., 1986. Best bounds for positive distributions with fixed moments. Insurance: Mathematics \& Economics 5, 87-92.

Makarov, G.D., 1981. Estimates for the distribution function of the sum of two random variables when the marginal distributions are fixed. Theory of Probability and its Applications 26, 803-806.

Nelsen, R.B., 1999. An Introduction to Copulas. Lecture Notes in Statistics, no. 139. Springer, New York.

Rüschendorf, L., 1982. Random variables with maximum sums. Advances in Applied Probability 14, 623-632.

Scaillet, O., 2005. A Kolmogorov-Smirnov type test for positive quadrant dependence. The Canadian Journal of Statistics 33 (3), $415-427$.

Sklar, A., 1959. Foncions de répartition à n dimensions et leurs marges. Publications de l'Institut de statistique de l'Université de Paris 8 , 229-231.

Williamson, R., Downs, T., 1990. Probabilistic arithmetic. I. Numerical methods for calculating convolutions and dependency bounds. International Journal of Approximate Reasoning 4, 89-158. 